Pacific

Journal of

Mathematics

\title{
CYCLIC PURSUIT ON COMPACT MANIFOLDS
}

DMITRI GEKHTMAN

Volume 289 No. 1

July 2017 


\title{
CYCLIC PURSUIT ON COMPACT MANIFOLDS
}

\author{
DMITRI GEKHTMAN
}

\begin{abstract}
We study a form of cyclic pursuit on Riemannian manifolds with positive injectivity radius. We conjecture that on a compact manifold, the piecewise geodesic loop formed by connecting consecutive pursuit agents either collapses to a point in finite time or converges to a closed geodesic. The main result is that this conjecture is valid for nonpositively curved compact manifolds.
\end{abstract}

\section{Introduction}

Our starting point is the classical three bug problem, first posed by Edouard Lucas [1877]: Three bugs start on the corners of an equilateral triangle, and each chases the next at unit speed. What happens? Answer: The bugs wind around the center of the triangle infinitely many times as they head inward along logarithmic spirals. They collide at the center of the triangle in finite time. We get similar behavior in general for a system of $n$ bugs starting at the vertices of a regular $n$-gon, each chasing its clockwise neighbor at unit speed; see, e.g., [Behroozi and Gagnon 1979]. For illustrations of cyclic pursuit with initial conditions on a regular $n$-gon, see clips 1 through 4 at http://tinyurl.com/Gekhtman-bugs. For details on the history of various versions of the $n$ bug problem, see the introduction of [Richardson 2001b].

Next, consider $n$ bugs starting at arbitrary positions in $\mathbb{R}^{d}$, with bug $i$ chasing bug $i+1 \bmod n$ at unit speed. Clips 5 through 7 at the address below demonstrate cyclic pursuit with randomly chosen initial conditions in the unit cube of $\mathbb{R}^{3}$. The typical observed behavior is as follows: Starting from the initial random configuration, chains of closely spaced bugs form, the chains come together to form a close approximation of a smooth knot, the knot unknots into an approximately circular loop, and the loop collapses to a point in finite time. The evolution of the piecewise linear loop connecting the bugs qualitatively resembles the curve-shortening flow on the space of smooth loops in $\mathbb{R}^{3}$. Richardson [2001b; 2001a] analyzed aspects of cyclic pursuit in $\mathbb{R}^{d}$. He showed that for $n \geq 7$, the only stable configuration of $n$ bugs in cyclic pursuit is a planar regular $n$-gon [2001a]. Based on simulations, Richardson conjectured that, if the initial positions of $n$ bugs are chosen uniformly

MSC2010: primary 53B21; secondary 37D40.

Keywords: cyclic pursuit, curve shortening, closed geodesics, nonpositive curvature. 
at random in $[0,1]^{d}$, the probability of converging asymptotically to the regular $n$-gon configuration approaches 1 as $n \rightarrow \infty$.

In this paper, we study cyclic pursuit on Riemannian manifolds with positive injectivity radius. To define pursuit in this case, we choose initial positions such that each bug is within the injectivity radius of the next, and we have each bug chase the next with velocity equal to the unit vector tangent to the shortest geodesic connecting it to the next bug. Unlike in the Euclidean case, the bugs do not necessarily all collide in finite time. Certainly, they cannot if the piecewise geodesic loop connecting consecutive bugs is not nullhomotopic. This leads to the conjecture that on a compact manifold, if the bugs do not collide in finite time, the loop connecting them converges to a closed geodesic. The main result of this paper is that the conjecture is valid for pursuit on manifolds of nonpositive curvature. Clips 8 and 9 demonstrate convergence on a flat Möbius band and a flat torus, respectively. Numerical simulations suggest the conjecture is valid in general. Clip 10 demonstrates cyclic pursuit on $S^{2}$ and clips 11 and 12 show pursuit on $\mathbb{R} \mathbb{P}^{2}$.

The organization of the paper is as follows: In Section 3, we study basic properties of cyclic pursuit in Euclidean space. In Section 4, we introduce cyclic pursuit on Riemannian manifolds. In Section 5, we prove a result which states roughly that, if the bugs enter a convex subset of a manifold, they stay in that subset. We derive as a consequence a condition for the pursuit to end in finite time. In Section 6, we prove subsequential convergence of the loop of bugs to a closed geodesic, and we obtain another criterion for pursuit to end in finite time. In Section 7 , we give a condition for the loop of bugs to converge to a closed geodesic. In Section 8, we discuss convergence to closed geodesics which are locally length-minimizing, in the sense that any other loop uniformly close to the geodesic is longer. Then, we prove our main result: for pursuit on a nonpositively curved compact manifold, the loop of bugs either collapses to a point in finite time or converges to a closed geodesic.

\section{Notation}

Unless otherwise stated, geodesics are parametrized at constant speed.

If $(M,\langle\cdot, \cdot\rangle)$ is a Riemannian manifold and $p, q \in M$ are connected by a unique shortest geodesic, $[p, q]$ denotes the shortest geodesic from $p$ to $q$, parametrized as a map from the unit interval $[0,1]$. Unless otherwise stated, $\|\cdot\|: T_{p} M \rightarrow \mathbb{R}$ denotes the norm associated to $\langle\cdot, \cdot\rangle_{p}$, and $d: M \times M \rightarrow \mathbb{R}$ denotes the distance function associated to the metric. We identify $S^{1}$ with $\mathbb{R} / \mathbb{Z}$. For each $x \in S^{1}$, we define $T_{x}: \mathbb{R} / \mathbb{Z} \rightarrow \mathbb{R} / \mathbb{Z}$ as the translation $T_{x}(s)=s+x$. If $\alpha, \gamma: S^{1} \rightarrow M$ are two loops, $d(\alpha, \gamma)=\sup _{s \in S^{1}} d(\alpha(s), \gamma(s))$ denotes the supremum distance. If $p \in M$ and $r>0$, $B_{r}(p)$ denotes the metric ball of radius $r$ centered at $p$. For $p \in M$ and $K \subset M$, $d(p, K)$ denotes $\inf _{q \in K} d(p, q)$. If $\alpha: S^{1} \rightarrow M$ is a closed geodesic and $\delta>0$, $\bar{N}_{\delta}(\alpha)=\left\{p \in M \mid d\left(p, \alpha\left(S^{1}\right)\right) \leq \delta\right\}$ denotes the closed $\delta$-neighborhood of $\alpha\left(S^{1}\right)$. 


\section{Cyclic pursuit in Euclidean space}

We define cyclic pursuit of $n$ bugs in $\mathbb{R}^{d}$ with initial positions $\left\{b_{i}(0\}_{i \in \mathbb{Z} / n}\right.$ as the unique collection of piecewise smooth functions $\left\{b_{i}:[0, \infty) \rightarrow \mathbb{R}^{n}\right\}_{i \in \mathbb{Z} / n}$ with the given initial conditions satisfying

(1) If $b_{i}(t) \neq b_{i+1}(t)$, then

$$
\dot{b}_{i}(t)=\frac{b_{i+1}(t)-b_{i}(t)}{\left\|b_{i+1}(t)-b_{i}(t)\right\|} .
$$

(2) If $b_{i}\left(t_{0}\right)=b_{i+1}\left(t_{0}\right)$, then $b_{i}(t)=b_{i+1}(t)$ for all $t>t_{0}$.

(3) If $b_{i}\left(t_{0}\right)=b_{0}\left(t_{0}\right)$ for all $i$, then $b_{i}(t)=b_{0}\left(t_{0}\right)$ for all $t>t_{0}$.

The following result is well known. We include a proof, as it will be useful later.

Proposition 3.1. For any set of initial conditions $\left\{b_{i}(0)\right\}_{i \in \mathbb{Z} / n}$, cyclic pursuit in $\mathbb{R}^{d}$ ends in finite time, i.e., there is a $t_{0}>0$ so that $b_{i}(t)=b_{0}\left(t_{0}\right)$ for all $i$ and all $t \geq t_{0}$.

Proof. Let $l_{i}(t)=d\left(b_{i}(t), b_{i+1}(t)\right)$, and let $l(t)=\sum_{i=1}^{n} l_{i}(t)$ be the length of the piecewise linear loop connecting the $b_{i}(t)$. We recall the following fact, which can be verified by direct computation: Fix $p \in \mathbb{R}^{d}$ and consider the function $d_{p}: \mathbb{R}^{d} \backslash\{p\} \rightarrow \mathbb{R}, d_{p}(q)=d(p, q)$. Then the gradient of $d_{p}$ at $q$ is the unit vector $(q-p) /\|q-p\|$.

Let $u_{i}=\left(b_{i+1}(t)-b_{i}(t)\right) /\left\|b_{i+1}(t)-b_{i}(t)\right\|$. Assuming for now that $l_{i}(t)>0$ for all $i$, we get

$$
\begin{aligned}
\frac{d}{d t} l_{i}(t) & =\left\langle u_{i}(t), \dot{b}_{i+1}(t)\right\rangle+\left\langle-u_{i}(t), \dot{b}_{i}(t)\right\rangle \\
& =\left\langle u_{i}(t), u_{i+1}(t)\right\rangle+\left\langle-u_{i}(t), u_{i}(t)\right\rangle=\cos \theta_{i}(t)-1,
\end{aligned}
$$

where $\theta_{i}(t) \in[0, \pi]$ is the angle between $u_{i}(t)$ and $u_{i+1}(t)$. By a theorem of Borsuk [1947], the sum of the exterior angles of a piecewise linear loop in $\mathbb{R}^{d}$ is at least $2 \pi$. So some $\theta_{i}$ is at least $\frac{2 \pi}{n}$, and we find that $\frac{d}{d t} l(t) \leq \cos \frac{2 \pi}{n}-1$. In other words, $\frac{d}{d t} l(t)$ is negative, with absolute value bounded below by $1-\cos \frac{2 \pi}{n}$. If some $l_{i}(t)$ is 0 , this effectively reduces $n$, so we still have the same bound on $\frac{d}{d t} l(t)$. Thus, pursuit ends by time $l(0)\left[1-\cos \frac{2 \pi}{n}\right]^{-1}$.

Remark. If the $\theta_{i}$ are all $\frac{\pi}{2}$ or less, then Jensen's inequality applied to $1-\cos \theta$ on $\left[0, \frac{\pi}{2}\right]$ yields $\left|\frac{d}{d t} l(t)\right| \geq n\left(1-\cos \frac{2 \pi}{n}\right)$. On the other hand, if at least one of the $\theta_{i}$ is greater than $\frac{\pi}{2}$, then $\left|\frac{d}{d t} l(t)\right| \geq 1-\cos \frac{\pi}{2}=1$. Thus, assuming that $l_{i}(t)>0$ for all $i$, we have $\left|\frac{d}{d t} l(t)\right| \geq \min \left[1, n\left(1-\cos \frac{2 \pi}{n}\right)\right]$. Since $\min \left[1, n\left(1-\cos \frac{2 \pi}{n}\right)\right]$ is a nonincreasing function of $n \geq 2$, we still have $\left|\frac{d}{d t} l(t)\right| \geq \min \left[1, n\left(1-\cos \frac{2 \pi}{n}\right)\right]$ if some (but not all) of the $l_{i}(t)$ are 0 . Hence, the time from the start of the pursuit process to its end is bounded above by $l(0) \cdot\left(\min \left[1, n\left(1-\cos \frac{2 \pi}{n}\right)\right]\right)^{-1}$, which grows linearly in $n$. (Compare this to the $O\left(n^{2}\right)$ bound on the time obtained from 
the estimate $\left|\frac{d}{d t} l(t)\right| \geq 1-\cos \frac{2 \pi}{n}$ in the last paragraph.) Note also that, in the case that the $b_{i}(0)$ are vertices of a regular planar $n$-gon, we get that the time to mutual capture is precisely $l(0) \cdot\left[n\left(1-\cos \frac{2 \pi}{n}\right)\right]^{-1}$.

\section{Pursuit on Riemannian manifolds}

Cyclic pursuit on a Riemannian manifold is defined just as in the Euclidean case: each bug's velocity is the unit vector pointing towards the next bug along the shortest geodesic connecting the two. To ensure that there is a unique shortest geodesic connnecting each pair of bugs, we consider only manifolds with positive injectivity radius, and we choose initial positions so that the distance between each bug and its prey is less than the injectivity radius.

Let $(M, g)$ be a manifold with positive injectivity radius denoted by $\operatorname{inj}(M)$, and let $\left\{b_{i}(0)\right\}_{i \in \mathbb{Z} / n}$ be initial positions in $M$ satisfying $d\left(b_{i}(0), b_{i+1}(0)\right)<\operatorname{inj}(M)$. Then we define $\left\{b_{i}:[0, \infty) \rightarrow M\right\}_{i \in \mathbb{Z} / n}$ as the unique collection of piecewise smooth functions with the given initial conditions satisfying

(1) If $b_{i}(t) \neq b_{i+1}(t)$, then

$$
\dot{b}_{i}(t)=\frac{\exp _{b_{i}(t)}^{-1}\left(b_{i+1}(t)\right)}{\left\|\exp _{b_{i}(t)}^{-1}\left(b_{i+1}(t)\right)\right\|} .
$$

(2) If $b_{i}\left(t_{0}\right)=b_{i+1}\left(t_{0}\right)$, then $b_{i}(t)=b_{i+1}(t)$ for all $t>t_{0}$.

(3) If $b_{i}\left(t_{0}\right)=b_{0}\left(t_{0}\right)$ for all $i$, then $b_{i}(t)=b_{0}\left(t_{0}\right)$ for all $t>t_{0}$.

Let $l_{i}(t)=d\left(b_{i}(t), b_{i+1}(t)\right)$. To see that the pursuit process is well defined for all $t \geq 0$, we need to check that each $l_{i}(t)$ is nonincreasing and thus stays less than $\operatorname{inj}(M)$.

To compute $\frac{d}{d t} l_{i}(t)$, we recall the following fact, which follows from the Gauss lemma of Riemannian geometry: If $p \in M$ and $U$ is a normal neighborhood of $p$, consider the function $d_{p}: U \backslash\{p\} \rightarrow \mathbb{R}$ given by $d_{p}(q)=d(p, q)$. The gradient of $d_{p}$ at $q$ is the tangent at $q$ of the shortest unit speed geodesic going from $p$ to $q$.

Now, if $l_{i}(t)>0$, we use the above fact and the law of motion (2) to compute, just as in the last section, that

$$
\frac{d}{d t} l_{i}(t)=\cos \theta_{i}(t)-1,
$$

where $\theta_{i}$ is the angle at $b_{i+1}(t)$ between $\left[b_{i}(t), b_{i+1}(t)\right]$ and $\left[b_{i+1}(t), b_{i+2}(t)\right]$. So if $l_{i}(t)>0$, then $\frac{d}{d t} l_{i}(t) \leq 0$ and thus $l_{i}$ is locally nonincreasing at $t$. On the other hand, if $l_{i}(t)=0$, then $l_{i}\left(t^{\prime}\right)=0$ for all $t^{\prime}>t$. So each $l_{i}$ is indeed nonincreasing, and the pursuit process is well defined.

For each $t \geq 0, i \in \mathbb{Z} / n$, let $\beta_{i}^{t}=\left[b_{i}(t), b_{i+1}(t)\right]$ be the shortest geodesic connecting $b_{i}(t)$ to $b_{i+1}(t)$. Let $\beta^{t}: \mathbb{R} / \mathbb{Z} \rightarrow M$ be the constant-speed piecewise 
geodesic loop formed by concatenating the $\beta_{i}^{t}$, with $\beta^{t}(0)=\beta^{t}(1)=b_{0}(t)$. Then $t \mapsto \beta^{t}$ is a homotopy of loops, so if $\beta^{0}$ is not nullhomotopic, the pursuit process will not end in finite time. So Proposition 3.1 does not generalize to pursuit on Riemannian manifolds. This leads to the following conjecture for compact manifolds:

Conjecture 4.1. If $M$ is a compact Riemannian manifold, and $\left\{b_{i}(0)\right\}_{i \in \mathbb{Z} / n}$ are initial conditions for cyclic pursuit, then the associated family of loops $\beta^{t}$ either collapses to a point in finite time or converges to a closed geodesic as $t \rightarrow \infty$.

By convergence above, we mean convergence in the quotient of $C^{0}\left(S^{1}, M\right)$ by rotations in the domain. In other words, a sequence of loops $\left\{\gamma_{j}\right\}_{j=1}^{\infty}$ converges to $\gamma: \mathbb{R} / \mathbb{Z} \rightarrow M$ if

$$
\lim _{j \rightarrow \infty} \inf _{c \in \mathbb{R}} \sup _{s \in \mathbb{R} / \mathbb{Z}} d\left(\gamma_{j}(s), \gamma(s+c)\right)=0 .
$$

We prove Conjecture 4.1 in the case of pursuit on nonpositively curved compact manifolds in Section 8.

Remark. As observed above, if pursuit ends in finite time, then $\beta^{0}$ is nullhomotopic. The converse is not true. For instance, suppose $\alpha$ is a nullhomotopic closed geodesic along which all sectional curvatures are negative, e.g., the neck of a dumbbell. We will see in Section 8 that if $\beta^{0}$ is sufficiently close to $\alpha$, then $\beta^{t}$ will converge to $\alpha$.

\section{Convex submanifolds}

We will need the following result, which states roughly that, if at some time the $b_{i}$ all belong to a convex set $K \subset M$, then they stay in $K$.

Proposition 5.1. Let $M^{d}$ be a Riemannian manifold with $\operatorname{inj}(M)>0,\left\{b_{i}(t)\right\}_{i \in \mathbb{Z} / n}$ cyclic pursuit curves on $M, l_{i}(t)=d\left(b_{i}(t), b_{i+1}(t)\right)$. Let $K^{d} \subset M$ be a smoothly embedded submanifold with boundary, topologically closed in $M$. Suppose there is an $R \in(0, \operatorname{inj}(M))$ so that for any two points $p_{1}, p_{2} \in K$ with $d\left(p_{1}, p_{2}\right)<R$, the geodesic segment $\left[p_{1}, p_{2}\right]$ is contained in $K$. If for some $t_{0} \in[0, \infty)$, all of the $b_{i}\left(t_{0}\right)$ are in $K$ and all of the $l_{i}\left(t_{0}\right)$ are less than $R$, then $b_{i}(t) \in K$ for all $i \in \mathbb{Z} / n$ and for all $t \geq t_{0}$.

Proof idea. If one of the bugs reaches $\partial K$, then, by the convexity assumption, the bug's velocity will not point out of $K$. So the bug will stay in $K$.

Proof. Since $K$ is closed, embedded, and of the same dimension as $M$, its topological boundary in $M$ is the boundary manifold $\partial K$. Suppose for the sake of contradiction that there is a $t_{1}>t_{0}$ and $j \in \mathbb{Z} / n$ so that $b_{j}\left(t_{1}\right)$ is not in $K$. Set

$$
t^{\prime}=\sup \left\{t \in\left[t_{0}, t_{1}\right] \mid b_{i}(t) \in K \text { for all } i\right\} \quad \text { and } \varepsilon=t_{1}-t^{\prime} .
$$


Then since $K$ is closed and the $b_{i}$ are continuous, all of the $b_{i}\left(t^{\prime}\right)$ are in $K$, and at least one of the $b_{i}\left(t^{\prime}\right)$ is in $\partial K$. Furthermore, for all $t \in\left(t^{\prime}, t^{\prime}+\varepsilon\right]$, at least one of the $b_{i}(t)$ is in $M \backslash K$.

For each $i \in \mathbb{Z} / n$, let $\left(V_{i}, x_{i}^{1}, \ldots, x_{i}^{d}\right)$ be a coordinate neighborhood of $b_{i}\left(t^{\prime}\right)$ with the property that $V_{i} \cap K=\left\{p \in V_{i} \mid x_{i}^{d}(p) \leq 0\right\}$, thus that $V_{i} \cap \partial K=\left\{p \in V_{i} \mid x_{i}^{d}(p)=0\right\}$. (If $b_{i}\left(t^{\prime}\right)$ is in the interior of $K$, it may be that $V_{i} \cap \partial K=\varnothing$ and $x_{i}^{d}<0$ on all of $V_{i}$.) Shrinking $\varepsilon$ if necessary, we may assume $b_{i}\left(\left[t^{\prime}, t^{\prime}+\varepsilon\right]\right) \subset V_{i}$ for all $i$. Let $b_{i}^{d}=x_{i}^{d} \circ b_{i}$ denote the $d$-th component of $b_{i}$. Let $h(t)=\max _{i} b_{i}^{d}(t)$. Since all of the $b_{i}\left(t^{\prime}\right)$ are in $K$, and at least one of $b_{i}\left(t^{\prime}\right)$ is in $\partial K, h\left(t^{\prime}\right)=0$. For each $t \in\left(t^{\prime}, t^{\prime}+\varepsilon\right]$, at least one of the $b_{i}(t)$ is in the complement of $K$, so $h(t)>0$. Assume without loss of generality that $b_{i}\left(t^{\prime}\right) \neq b_{i+1}\left(t^{\prime}\right)$, for all $i$. Then take $\varepsilon$ small enough that $b_{i}(t) \neq b_{i+1}(t)$, for all $t \in\left[t^{\prime}, t^{\prime}+\varepsilon\right]$ and all $i \in \mathbb{Z} / n$. Since each $b_{i}^{d}$ is smooth on $\left[t^{\prime}, t^{\prime}+\varepsilon\right], h$ is absolutely continuous on $\left[t^{\prime}, t^{\prime}+\varepsilon\right]$. So $h$ is almost everywhere differentiable, and

$$
h(t)=\int_{t^{\prime}}^{t} \frac{d}{d s} h(s) d s
$$

for each $t \in\left[t^{\prime}, t^{\prime}+\varepsilon\right]$. Thus, for some $c_{1} \in[0, \varepsilon], \frac{d}{d t} h\left(t^{\prime}+c_{1}\right)$ is defined and

$$
h\left(t^{\prime}+\varepsilon\right) \leq \varepsilon \frac{d}{d t} h\left(t^{\prime}+c_{1}\right)=\varepsilon \frac{d}{d t} b_{j}^{d}\left(t^{\prime}+c_{1}\right),
$$

for some $j$ for which $b_{j}^{d}\left(t^{\prime}+c_{1}\right)=h\left(t^{\prime}+c_{1}\right)$. Take $\varepsilon$ small enough that if $b_{i}^{d}(t)=h(t)$ for some $t \in\left[t^{\prime}, t^{\prime}+\varepsilon\right], b_{i}^{d}\left(t^{\prime}\right)=0$. Then in particular, $b_{j}^{d}\left(t^{\prime}\right)=0$ for the $j$ in the last displayed formula.

For each $i \in \mathbb{Z} / n$, let $v_{i}^{d}(p, q)$ be the $d$-th component of the initial unit tangent to $[p, q]$, for $(p, q) \in V_{i} \times V_{i+1}$ with $0<d(p, q)<\operatorname{inj}(M)$. By the law of motion (2),

$$
\frac{d}{d t} b_{j}^{d}\left(t^{\prime}+c_{1}\right)=v_{j}^{d}\left(b_{j}\left(t^{\prime}+c_{1}\right), b_{j+1}\left(t^{\prime}+c_{1}\right)\right) .
$$

For each $i$, let $B_{i} \subset V_{i}$ be an open coordinate ball centered at $b_{i}\left(t^{\prime}\right)$ with $\bar{B}_{i} \subset V_{i}$. Shrinking the $B_{i}$ if necessary, assume there is a $\delta>0$ so that $\delta<d(p, q)<R$ for all $(p, q) \in B_{i} \times B_{i+1}$. Then $v^{d}$ is $C^{1}$ on $\bar{B}_{i} \times \bar{B}_{i+1}$. Since $K$ contains $[p, q]$ whenever $p, q \in K$ and $d(p, q)<R$, we have for all $(p, q) \in B_{i} \times B_{i+1}$ with $x_{i}^{d}(p)=0$ and $x_{i+1}^{d}(q) \leq 0$, that $v_{i}^{d}(p, q) \leq 0$. Let $\mu_{i}$ and $v_{i}$ be the maximum on $\bar{B}_{i} \times \bar{B}_{i+1}$ of the absolute value of the derivative of $v_{i}^{d}(p, q)$ with respect to the $d$-th component of $p$ and $q$, respectively. Let $\mu=\max _{i} \mu_{i}$ and $v=\max _{i}=v_{i}$. Taking $\varepsilon$ small enough that $b_{i}\left(\left[t^{\prime}, t^{\prime}+\varepsilon\right]\right) \subset B_{i}$ for all $i$, we have

$$
v_{j}^{d}\left(b_{j}\left(t^{\prime}+c_{1}\right), b_{j+1}\left(t^{\prime}+c_{1}\right)\right) \leq(\mu+v) h\left(t^{\prime}+c_{1}\right) .
$$

From the last three displayed formulas, we get

$$
h\left(t^{\prime}+\varepsilon\right) \leq(\mu+v) \varepsilon h\left(t^{\prime}+c_{1}\right) .
$$


Similarly, $h\left(t^{\prime}+c_{1}\right) \leq(\mu+v) \varepsilon h\left(t^{\prime}+c_{2}\right)$ for some $c_{2} \in\left[0, c_{1}\right]$, so we obtain $h\left(t^{\prime}+\varepsilon\right) \leq((\mu+v) \varepsilon)^{2} h\left(t^{\prime}+c_{2}\right)$. Inductively, we get for each positive integer $k$,

$$
h\left(t^{\prime}+\varepsilon\right) \leq((\mu+v) \varepsilon)^{k} h\left(t^{\prime}+c_{k}\right)
$$

for some $c_{k} \in[0, \varepsilon]$. Let $C=\max _{i \in \mathbb{Z} / n} \sup _{p \in B_{i}}\left|x_{i}^{d}(p)\right|$. Taking $\varepsilon<\frac{1}{2}(\mu+v)^{-1}$, we get $h\left(t^{\prime}+\varepsilon\right)<2^{-k} C$. Letting $k \rightarrow \infty$ yields $h\left(t^{\prime}+\varepsilon\right)=0$, a contradiction.

We say a subset $K$ of a Riemannian manifold $M$ is convex if for each pair $p, q \in K$, there is a unique shortest geodesic in $M$ connecting $p, q$ and this geodesic is contained in $K$. Proposition 5.1 is the key ingredient in the proof of the following result:

Proposition 5.2. Suppose $M$ is compact and $\left\{b_{i}(t)\right\}_{i \in \mathbb{Z} / n}$ are cyclic pursuit curves in $M$. Let $l_{i}(t)=d\left(b_{i}(t), b_{i+1}(t)\right)$ for each $i$. If $l_{i}(t) \rightarrow 0$ for all $i$, then pursuit ends in finite time.

Proof idea. Reduce to the Euclidean case by noting that the bugs will eventually lie in a small, convex, approximately Euclidean ball.

Proof. Assume for the sake of contradiction that pursuit does not end in finite time. Without loss of generality, assume $l_{i}(t)>0$ for all $i$. Since $M$ is compact, there is a $p \in M$ and a sequence of times $\left(t_{j}\right)_{j=1}^{\infty}$ with $t_{j} \rightarrow \infty$ so that $b_{0}\left(t_{j}\right) \rightarrow p$ as $j \rightarrow \infty$. Let $r \in(0, \operatorname{inj}(M))$ be small enough that $\bar{B}_{r}(p)$, the closed $r$-ball centered at $p$, is convex. Since $d\left(b_{i}\left(t_{j}\right), b_{i+1}\left(t_{j}\right)\right) \rightarrow 0, b_{0}\left(t_{j}\right) \rightarrow p$, and $b_{i}\left(t_{j}\right) \rightarrow p$ for all $i$, there is a $J$ for which all of the $b_{i}\left(t_{J}\right)$ belong to $\bar{B}_{r}(p)$. By Proposition 5.1, the $b_{i}(t)$ remain in $\bar{B}_{r}(p)$ for all $t>t_{J}$.

Let $\left(U, x^{i}\right)$ be a normal coordinate neighborhood centered at $p$. By Corollary A.2, we have that for small enough $r, \bar{B}_{r}(p)$ is a convex subset of $U$ and has the following property: for any two geodesics $\gamma_{1}:\left[0, a_{1}\right] \rightarrow \bar{B}_{r}(p)$ and $\gamma_{2}:\left[0, a_{1}\right] \rightarrow \bar{B}_{r}(p)$ with $\gamma_{1}(0)=\gamma_{2}(0)$, the metric angle between $\dot{\gamma}_{1}(0)$ and $\dot{\gamma}_{2}(0)$ is within $\frac{\pi}{n}$ of the Euclidean angle, computed in the coordinates $\left(U, x^{i}\right)$, between $\gamma_{1}\left(a_{1}\right)-\gamma_{1}(0)$ and $\gamma_{2}\left(a_{2}\right)-\gamma_{2}(0)$.

Now, choose $r$ as above and find $t_{0}$ so that all of the $b_{i}(t)$ are in $\bar{B}_{r}(t)$ for $t \geq t_{0}$. As we showed in the proof of Proposition 3.1, at least one of the Euclidean angles of the piecewise linear loop connecting the $b_{i}(t)$ is at least $\frac{2 \pi}{n}$. So by the result quoted in the last paragraph, at least one of the angles of the piecewise geodesic loop connecting the $b_{i}(t)$ is at least $\frac{\pi}{n}$. Thus, $\frac{d}{d t} l(t) \leq \cos \frac{\pi}{n}-1$ for $t \geq t_{0}$ and so pursuit ends by time $t_{0}+l\left(t_{0}\right)\left(1-\cos \frac{\pi}{n}\right)^{-1}$. This is a contradiction.

\section{Subsequential convergence}

In this section, $M$ is a compact Riemannian manifold. 
Proposition 6.1. Let $\left\{b_{i}(t)\right\}_{i \in \mathbb{Z} / n}$ be pursuit curves on $M$, let $\beta^{t}$ be the associated family of piecewise geodesic loops, and let $l(t)$ the length of $\beta^{t}$. If the pursuit does not end in finite time, then there is a sequence of times $\left(t_{j}\right)_{j=1}^{\infty}, t_{j} \rightarrow \infty$ so that $\beta^{t_{j}}$ converges uniformly to a closed geodesic of length $L=\lim _{t \rightarrow \infty} l(t)$ as $j \rightarrow \infty$.

Proof sketch. Take a sequence $t_{j}$ so that the $b_{i}\left(t_{j}\right)$ converge and so that $\frac{d}{d t} l\left(t_{j}\right) \rightarrow 0$. Then $\beta^{t_{j}}$ converges to a piecewise geodesic loop. The condition $\frac{d}{d t} l\left(t_{j}\right) \rightarrow 0$ implies that the angles between segments of the limiting loop are 0 .

Proof. Let $\beta_{i}^{t}=\left[b_{i}(t), b_{i+1}(t)\right]$. Recall that $\beta^{t}$ is the constant-speed piecewise geodesic loop formed from the $\beta_{i}^{t}$, with $\beta^{t}(0)=b_{0}(t)$. We have $L>0$, else by Proposition 5.2, pursuit ends in finite time. Assume without loss of generality that $d\left(b_{i}(t), b_{i+1}(t)\right)>0$ for all $i \in \mathbb{Z} / n$ and all $t>0$. Then we have for all $t$ that

$$
\frac{d}{d t} l(t)=\sum_{i}\left(\cos \theta_{i}(t)-1\right)
$$

where $\theta_{i}(t)$ is the angle at $b_{i+1}(t)$ between $\beta_{i}^{t}$ and $\beta_{i+1}^{t}$. Since $l$ is differentiable, nonincreasing, and bounded from below, there is a sequence $\left(t_{j}\right)_{j=1}^{\infty}, t_{j} \rightarrow \infty$, so that $\frac{d}{d t} l\left(t_{j}\right) \rightarrow 0$. This implies that for each $i, \theta_{i}\left(t_{j}\right) \rightarrow 0$ as $j \rightarrow \infty$. Since $M$ is compact, we may pass to a subsequence and assume that for each $i, b_{i}\left(t_{j}\right)$ converges to some point $a_{i} \in M$. Then $\left[b_{i}\left(t_{j}\right), b_{i+1}\left(t_{j}\right)\right]$ converges uniformly to $\left[a_{i}, a_{i+1}\right]$. Let $\alpha$ be the constant speed piecewise geodesic loop formed from the geodesic segments $\left[a_{i}, a_{i+1}\right]$, with $\alpha(0)=a_{0}$. Then $\beta^{t_{j}}$ converges to $\alpha$ uniformly. By continuity, $\alpha$ has length $L$.

We need to show that $\alpha$ is a closed geodesic. To do this, it suffices to show that the angles between successive geodesic segments comprising $\alpha$ are 0 . We need to include the case that $a_{i}=a_{i+1}$ for some $i$. To this end, suppose $a_{i-1} \neq a_{i}$, and let $k$ be the largest integer so that $a_{i}=a_{i+1}=\cdots=a_{i+k}$. We need to show that the angle at $a_{i}$ between $\left[a_{i-1}, a_{i}\right]$ and $\left[a_{i}, a_{i+k+1}\right]$ is 0 . Let $\left(U, x^{i}\right)$ be a normal coordinate neighborhood centered at $a_{i}$, and $\|\cdot\|_{U}$ be the Euclidean norm on $T U$ coming from the coordinates. Fix $\varepsilon>0$. Then for large enough $j, b_{i}\left(t_{j}\right), \ldots, b_{i+k}\left(t_{j}\right)$ are in $U$ and

$$
\left\|\frac{\dot{\beta}_{m}^{t_{j}}(1)}{\left\|\dot{\beta}_{m}^{t_{j}}(1)\right\|}-\frac{\dot{\beta}_{m}^{t_{j}}(0)}{\left\|\dot{\beta}_{m}^{t_{j}}(0)\right\|}\right\|_{U}<\varepsilon,
$$

for $m=i, \ldots, i+k-1$. (See (9) in the Appendix.) Since $\theta_{i}\left(t_{j}\right) \rightarrow 0$ for all $i$, for large enough $j$,

$$
\left\|\frac{\dot{\beta}_{m}^{t_{j}}(0)}{\left\|\dot{\beta}_{m}^{t_{j}}(0)\right\|}-\frac{\dot{\beta}_{m-1}^{t_{j}}(1)}{\left\|\dot{\beta}_{m-1}^{t_{j}}(1)\right\|}\right\|_{U}<\varepsilon
$$


for $m=i, \ldots, i+k$. From the last two displayed expressions, we obtain

$$
\left\|\frac{\dot{\beta}_{i+k}^{t_{j}}(0)}{\left\|\dot{\beta}_{i+k}^{t_{j}}(0)\right\|}-\frac{\dot{\beta}_{i-1}^{t_{j}}(1)}{\left\|\dot{\beta}_{i-1}^{t_{j}}(1)\right\|}\right\|_{U}<(2 k-1) \varepsilon .
$$

Thus, the expression on the left of the last inequality converges to 0 as $j \rightarrow \infty$. But

$$
\frac{\dot{\beta}_{i+k}^{t_{j}}(0)}{\left\|\dot{\beta}_{i+k}^{t_{j}}(0)\right\|}
$$

converges to the unit tangent to $\left[a_{i}, a_{i+k+1}\right]$ at $a_{i}$, and

$$
\frac{\dot{\beta}_{i-1}^{t_{j}}(1)}{\left\|\dot{\beta}_{i-1}^{t_{j}}(1)\right\|}
$$

converges to the unit tangent to $\left[a_{i-1}, a_{i}\right]$ at $a_{i}$. Hence, these two unit tangent directions are the same, and the angle between $\left[a_{i}, a_{i+k+1}\right]$ and $\left[a_{i-1}, a_{i}\right]$ at $a_{i}$ is 0 , as claimed.

As a consequence of the last proposition, we have the following corollaries.

Corollary 6.2. If for some $t_{0} \geq 0$, the length of $\beta^{t_{0}}$ is less than the length $\lambda_{\min }$ of the shortest closed geodesic of $M$, pursuit ends in finite time. In particular, if the length of $\beta^{0}$ is less than $\lambda_{\min }$, pursuit ends in finite time.

Corollary 6.3. If for some $t_{0} \geq 0$, the $b_{i}\left(t_{0}\right)$ all lie in a convex, smoothly embedded, closed metric ball $\bar{B} \subset M$, then pursuit ends in finite time.

Proof. By Proposition 5.1, the $b_{i}(t)$ stay in $\bar{B}$ for $t>t_{0}$. If pursuit does not end in finite time, then arguing as in Proposition 6.1, there is a sequence $t_{j} \rightarrow \infty$ so that $\beta^{t_{j}}$ converges to a closed geodesic in $\bar{B}$. But there are no closed geodesics contained in $\bar{B}$.

It follows, for example, that if the $b_{i}\left(t_{0}\right)$ all lie in an open hemisphere of $S^{2}$ with its standard metric, pursuit ends in finite time.

\section{A criterion for convergence}

The next result gives a criterion for convergence of $\beta^{t}$ to a closed geodesic $\alpha$.

Proposition 7.1. Let $M$ be a Riemannian manifold with $\operatorname{inj}(M)>0$. Let $\left\{b_{i}(t)\right\}_{i \in \mathbb{Z} / n}$ be cyclic pursuit curves on $M$, and let $\beta^{t}$ the associated family of loops. Suppose there is a sequence $t_{j} \rightarrow \infty$ and a closed geodesic $\alpha$ so that $\beta^{t_{j}} \rightarrow \alpha$ uniformly. If $\sup _{s \in S^{1}} d\left(\beta^{t}(s), \alpha\left(S^{1}\right)\right) \rightarrow 0$ as $t \rightarrow \infty$, then $\beta^{t}$ converges to $\alpha$ in the sense of (3), as $t \rightarrow \infty$. 
Proof idea. For large $t$, the curves $\beta^{t}$ and $\alpha$ have approximately the same length. In addition, $\beta^{t}$ fits into a small tubular neighborhood of $\alpha$. These two facts force $\beta^{t}$ to be uniformly close to $\alpha$.

Proof. Let $U$ be an open neighborhood of $\alpha\left(S^{1}\right)$ such that each $p \in U$ has a unique closest point $\pi(p)$ on $\alpha\left(S^{1}\right)$. Shrinking $U$ if necessary, we may construct a smooth unit vector field $X$ on $U$ extending the unit tangent field $\dot{\alpha} /\|\dot{\alpha}\|$ of $\alpha$.

Fix $\varepsilon>0$. Let $l_{i}(t)=d\left(b_{i}(t), b_{i+1}(t)\right)$, and let $\lambda$ be the minimum of $\lim _{t \rightarrow \infty} l_{i}(t)$ over $i$ for which $\lim _{t \rightarrow \infty} l_{i}(t)>0$. The continuous dependence of the initial unit tangent of a geodesic [ $p, q]$ on the endpoints $p$ and $q$ implies: There is a $\delta$ such that $\bar{N}_{\delta}(\alpha) \subset U$ and if $\gamma:[0,1] \rightarrow \bar{N}_{\delta}(\alpha)$ is a geodesic of length at least $\lambda$, then the component of $\dot{\gamma}(0) /\|\dot{\gamma}(0)\|$ normal to $X(\gamma(0))$ has length less than $\varepsilon$.

Consider $i$ such that $\lim _{t \rightarrow \infty} l_{i}(t)>0$. Let $a_{i}^{t}=\pi\left(\beta_{i}^{t}(0)\right)$. By hypothesis,

$$
d\left(\beta_{i}^{t}(0), a_{i}^{t}\right) \rightarrow 0
$$

as $t \rightarrow \infty$. By the observation in the previous paragraph, the component of $\dot{\beta}_{i}^{t}(0)$ orthogonal to $X\left(\beta_{i}^{t}(0)\right)$ goes to 0 as well. Since $\beta^{t_{j}} \rightarrow \alpha$ uniformly, we have by continuity that

$$
\left\|\dot{\beta}_{i}^{t}(0)-l_{i}(t) \cdot X\left(\beta_{i}^{t}(0)\right)\right\| \rightarrow 0 .
$$

Now, let $\alpha_{i}^{t}$ be the segment of $\alpha$ starting at $a_{i}^{t}$ with initial velocity $l_{i}(t) \cdot X\left(a_{i}(t)\right)$. Since a geodesic depends continuously on its initial parameters, (4) and (5) give

$$
\lim _{t \rightarrow \infty} \sup _{s \in[0,1]} d\left(\beta_{i}^{t}(s), \alpha_{i}^{t}(s)\right)=0 .
$$

Let $i_{1}, \ldots, i_{m}$ be the values of $i$ for which $\lim _{t \rightarrow \infty} l_{i}(t)>0$, listed in order. Now, let $\gamma^{t}: \mathbb{R} / \mathbb{Z} \rightarrow M$ be the piecewise continuous loop formed by concatenating the segments $\alpha_{i_{j}}^{t}([0,1))$. We parametrize $\gamma^{t}$ so that each $\alpha_{i_{j}}^{t}$ is traversed at the same constant speed, and $\gamma^{t}(0)=\alpha_{i_{1}}^{t}(0)$. Then as a consequence of (6),

$$
\lim _{t \rightarrow \infty} \sup _{s \in S^{1}} d\left(\beta^{t}(s), \gamma^{t}(s)\right)=0 .
$$

Let $c^{t}$ be such that $\alpha\left(c^{t}\right)=\gamma^{t}(0)$. Then by applying the triangle inequality, $\lim _{t \rightarrow \infty} d\left(\alpha_{i_{j}}^{t}(1), \alpha_{i_{j+1}}^{t}(0)\right)=0$ for $j=1, \ldots, m-1$. Also, $\sum_{j=1}^{m} l_{i_{j}}(t)$ converges to the length of $\alpha$ as $t \rightarrow \infty$. It follows that

$$
\lim _{t \rightarrow \infty} \sup _{s \in S^{1}} d\left(\gamma^{t}(s), \alpha\left(s+c^{t}\right)\right)=0 .
$$

From (7) and (8), we get

$$
\lim _{t \rightarrow \infty} \sup _{s \in S^{1}} d\left(\beta^{t}(s), \alpha\left(s+c^{t}\right)\right)=0,
$$

which completes the proof. 


\section{Nonpositive curvature}

In the next proposition, we show that if a subsequence $\beta^{t_{j}}$ converges to a closed geodesic $\alpha$ which is a local minimizer of length, then $\beta^{t}$ converges to $\alpha$.

We recall the following notation: For each $x \in S^{1}$, let $T_{x}: \mathbb{R} / \mathbb{Z} \rightarrow \mathbb{R} / \mathbb{Z}$ be the translation $T_{x}(s)=s+x$. If $\alpha, \gamma: S^{1} \rightarrow M$ are two loops, $d(\alpha, \gamma)$ denotes the supremum distance $\sup _{s \in S^{1}} d(\alpha(s), \gamma(s))$.

Proposition 8.1. Let $M$ be a Riemannian manifold with $\operatorname{inj}(M)>0,\left\{b_{i}(t)\right\}_{i \in \mathbb{Z} / n}$ cyclic pursuit curves on $M, \beta^{t}$ the associated family of loops. Let $\alpha$ be a closed geodesic of length $L$, and suppose there is $\varepsilon>0$ so that any rectifiable, constant speed loop $\gamma$ of length $L$ with $d(\alpha, \gamma)<\varepsilon$ is a reparametrization of $\alpha$, i.e., $\gamma=\alpha \circ T_{x}$ for some $x \in S^{1}$. If there is a sequence of times $t_{j} \rightarrow \infty$ so that $\beta^{t_{j}} \rightarrow \alpha$ as $j \rightarrow \infty$, then $\beta^{t} \rightarrow \alpha$ as $t \rightarrow \infty$.

Proof sketch. If $\beta^{t}$ does not converge to $\alpha$, then $\beta^{t}$ has another length $L$ subsequential limit $\gamma$. It follows that for any $k$, there is a homotopy from $\alpha$ to $\gamma$ through curves of length between $L$ and $L+1 / k$. The homotopy passes through a curve $\eta_{k}$ so that $\inf _{x \in S^{1}} d\left(\eta_{k}, \alpha \circ T_{x}\right)=\frac{\varepsilon}{2}$. Taking a subsequential limit of the $\eta_{k}$ yields a contradiction.

Proof. Suppose for the sake of contradiction that $\beta^{t}$ does not converge to $\alpha$. Then by Proposition 7.1, there is $\delta>0$ and a sequence of times $t_{j}^{\prime} \rightarrow \infty$ so that $\sup _{s \in S^{1}} d\left(\beta^{t_{j}^{\prime}}(s), \alpha\left(S^{1}\right)\right)>\delta$. Passing to a subsequence, we may assume $\beta^{t_{j}^{\prime}}$ converges uniformly to a constant speed piecewise geodesic loop $\gamma$ as $j \rightarrow \infty$.

Since $\sup _{s \in S^{1}} d\left(\beta^{t_{j}^{\prime}}(s), \alpha\left(S^{1}\right)\right)>\delta$ for all $j, \sup _{s \in S^{1}} d\left(\gamma(s), \alpha\left(S^{1}\right)\right) \geq \delta$, so $\gamma$ is not a reparametrization of $\alpha$. But since $\beta^{t_{j}} \rightarrow \alpha, \lim _{t \rightarrow \infty} l(t)=L$, and thus $\gamma$ has length $L$. So by hypothesis, $d\left(\gamma \circ T_{x}, \alpha\right) \geq \varepsilon$ for all $x \in S^{1}$, which is to say that $\inf _{x \in S^{1}} d\left(\gamma \circ T_{x}, \alpha\right) \geq \varepsilon$. We may assume that $t_{j}<t_{j}^{\prime}, d\left(\beta^{t_{j}}, \alpha\right)<\frac{\varepsilon}{2}$, and $d\left(\beta^{t_{j}^{\prime}}, \gamma\right)<\frac{\varepsilon}{2}$ for all $j$. Then

$$
\inf _{x \in S^{1}} d\left(\beta^{t_{j}} \circ T_{x}, \alpha\right) \leq d\left(\beta^{t_{j}}, \alpha\right)<\frac{\varepsilon}{2},
$$

and

$$
\inf _{x \in S^{1}} d\left(\beta^{t_{j}^{\prime}} \circ T_{x}, \alpha\right) \geq\left(\inf _{x \in S^{1}} d\left(\gamma \circ T_{x}, \alpha\right)\right)-d\left(\beta^{t_{j}^{\prime}}, \gamma\right)>\frac{\varepsilon}{2} .
$$

Since the function $(t, x) \mapsto d\left(\beta^{t} \circ T_{x}, \alpha\right)$ is continuous and $S^{1}$ is compact, the function $t \mapsto \inf _{x \in S^{1}} d\left(\beta^{t} \circ T_{x}, \alpha\right)$ is continuous. Thus, for each $j$, there is $t_{j}^{\prime \prime} \in\left(t_{j}, t_{j}^{\prime}\right)$, so that $\inf _{x \in S^{1}} d\left(\beta^{t_{j}^{\prime \prime}} \circ T_{x}, \alpha\right)=\frac{\varepsilon}{2}$. A subsequence of $\beta^{t_{j}^{\prime \prime}}$ converges to a constant speed loop $\eta$ of length $L$ with

$$
\inf _{x \in S^{1}} d\left(\eta \circ T_{x}, \alpha\right)=\frac{\varepsilon}{2} .
$$


Hence, $\eta$ is not a reparametrization of $\alpha$, yet there is an $x$ so that $d\left(\eta \circ T_{x}, \alpha\right)=\frac{\varepsilon}{2}<\varepsilon$. This is a contradiction.

With the notation as above we have the following:

Corollary 8.2. Let $\alpha$ be a closed geodesic of length $L$ such that all sectional curvatures are negative at each point of the image of $\alpha$. If $\beta^{t_{j}} \rightarrow \alpha$ for some sequence $t_{j} \rightarrow \infty$, then $\beta^{t} \rightarrow \alpha$.

Proof. From the formula for second variation of arclength, we know that $\alpha$ is isolated in the space of loops of length $L$, i.e., that $\alpha$ satisfies the hypotheses of Proposition 8.1.

Remark. Suppose a subsequence $\beta^{t_{j}}$ converges to a closed geodesic $\alpha$. Then Proposition 8.1 shows that if $\alpha$ is isolated in the space of rectifiable loops of its length, then $\beta^{t} \rightarrow \alpha$. Suppose instead $\alpha$ is merely isolated in the space of closed geodesics of its length. Suppose in addition that $\theta_{i}(t)$ converges to 0 for all $i$. Then all subsequential limits of $\beta^{t}$ are geodesics, so arguing as in the proof of Proposition 8.1, we can show $\beta^{t} \rightarrow \alpha$. In particular, if the following conjecture holds, then $\beta^{t}$ converges to a closed geodesic (or a point) for pursuit on any compact manifold whose space of closed geodesics is discrete.

Conjecture 8.3. Let $M$ be a compact manifold, $\left\{b_{i}(t)\right\}_{i \in \mathbb{Z} / n}$ pursuit curves on $M$, $l_{i}(t)$ the associated lengths, $\theta_{i}(t)$ the associated angles. If $l_{i}(t)>0$ for all $i \in \mathbb{Z} / n$ and $t \geq 0$, then $\theta_{i}(t) \rightarrow 0$.

Corollary 8.2 and Proposition 6.1 imply Conjecture 4.1 for compact manifolds of negative curvature. Next, we prove Conjecture 4.1 for manifolds of nonpositive curvature. First, we need a lemma:

Lemma 8.4. Let $\alpha$ be a closed geodesic on a Riemannian manifold M. Suppose $\alpha\left(S^{1}\right)$ is contained in a nonpositively curved open submanifold $U \subset M$. Suppose further that there is an $r>0$ so that $\operatorname{inj}(p) \geq r$ for all $p \in U$. Fix $\varepsilon>0$. For sufficiently small $\delta$, the following property holds: for any $p_{1}, p_{2} \in \bar{N}_{\delta}(\alpha)$ with $d\left(p_{1}, p_{2}\right)<r-\varepsilon$, we have $\left[p_{1}, p_{2}\right] \subset \bar{N}_{\delta}(\alpha)$.

Proof. We will show that it suffices to take $\delta$ small enough that

(i) $\delta<\frac{\varepsilon}{4}$,

(ii) for any $p, q \in \bar{N}_{\delta}(\alpha)$ with $d(p, q)<r-\frac{\varepsilon}{4}$, we have $[p, q] \subset U$.

(For (ii), we use the continuous dependence of $[p, q]$ on $p, q$.)

Now, take $p_{1}, p_{2} \in \bar{N}_{\delta}(\alpha)$ with $d\left(p_{1}, p_{2}\right)<r-\varepsilon$. Let $\alpha:[0,1] \rightarrow M$ be the shortest geodesic connecting $p_{1}$ to $p_{2}$. Choose $q_{i}$ in the image of $\alpha$ with $d\left(p_{i}, q_{i}\right) \leq \delta$ for $i=1,2$. By condition (i) and the triangle inequality, $d\left(q_{1}, q_{2}\right)<r-\frac{\varepsilon}{2}$, so there is a unique shortest geodesic $\gamma:[0,1] \rightarrow M$ connecting $q_{1}$ to $q_{2}$. 
Observe that $d(\alpha(t), \gamma(t))<r-\frac{\varepsilon}{4}$ for all $t \in[0,1]$. Indeed, for $t \in[0,1 / 2]$, the path consisting of segments $\left[\alpha(t), p_{1}\right],\left[p_{1}, q_{1}\right],\left[q_{1}, \gamma(t)\right]$ has length less than $r-\frac{\varepsilon}{4}$. Similarly, for $t \in[1 / 2,1]$, the path consisting of $\left[\alpha(t), p_{2}\right],\left[p_{2}, q_{2}\right],\left[q_{2}, \gamma(t)\right]$ has length less than $r-\frac{\varepsilon}{4}$.

By (ii), it follows that $[\alpha(t), \gamma(t)] \subset U$ for $t \in[0,1]$. The fact that $d(\alpha(t), \gamma(t))<$ $r-\frac{\varepsilon}{4}$ for all $t \in[0,1]$ also implies that the geodesic $[\alpha(t), \gamma(t)]$ varies smoothly in $t$. Since $U$ is nonpositively curved, we may apply the formula for the second variation of energy to the family of geodesics $[\alpha(t), \gamma(t)]$ to conclude that $d^{2}(\alpha(t), \gamma(t))$ is convex as a function of $t$. Therefore, for $t \in[0,1]$,

$$
\begin{aligned}
d(\alpha(t), \gamma(t)) & \leq \max [d(\alpha(0), \gamma(0)), d(\alpha(1), \gamma(1))] \\
& =\max \left[d\left(p_{1}, q_{1}\right), d\left(p_{2}, q_{2}\right)\right] \leq \delta .
\end{aligned}
$$

Thus, $\left[p_{1}, p_{2}\right] \subset \bar{N}_{\delta}(\alpha)$.

Proposition 8.5. If pursuit on a nonpositively curved compact manifold $M$ does not end in finite time, there is a closed geodesic $\alpha$ so that $\sup _{s \in S^{1}} d\left(\beta^{t}(s), \alpha\left(S^{1}\right)\right) \rightarrow 0$ as $t \rightarrow \infty$.

Proof. By Proposition 6.1, there is a closed geodesic $\alpha$ and a sequence $t_{j} \rightarrow \infty$ so that $\beta^{t_{j}} \rightarrow \alpha$ uniformly.

Let $\varepsilon>0$ be such that $d\left(b_{i}(0), b_{i+1}(0)\right)<\operatorname{inj}(M)-\varepsilon$ for all $i$. Take $\delta<\frac{\varepsilon}{4}$. Then by the proof of Lemma 8.4, if $p_{1}, p_{2} \in \bar{N}_{\delta}(\alpha)$ and $d\left(p_{1}, p_{2}\right)<\operatorname{inj}(M)-\varepsilon$, we have $\left[p_{1}, p_{2}\right] \subset \bar{N}_{\delta}(\alpha)$. Also take $\delta$ small enough that $\bar{N}_{\delta}(\alpha)$ is a closed manifold with boundary, smoothly embedded in $M$. Since $\beta^{t_{j}} \rightarrow \alpha$, we have that the $b_{i}\left(t_{J}\right)$ are all in $\bar{N}_{\delta}(\alpha)$ for some sufficiently large $J$. Now, by Proposition 5.1, we have $b_{i}(t) \in \bar{N}_{\delta}(\alpha)$ for all $i \in \mathbb{Z} / n, t \geq t_{J}$. By Lemma 8.4, $\beta^{t} \subset \bar{N}_{\delta}(\alpha)$ for $t \geq t_{J}$. Thus, $\sup _{s \in S^{1}} d\left(\beta^{t}(s), \alpha\right) \rightarrow 0$ as $t \rightarrow \infty$.

As a consequence of Proposition 7.1 and Proposition 8.5, we have Conjecture 4.1 for manifolds of nonpositive curvature:

Theorem 8.6. Let $M$ be a compact manifold of nonpositive sectional curvature. Suppose pursuit on $M$ with initial positions $\left\{b_{i}(0)\right\}_{i \in \mathbb{Z} / n}$ does not end in finite time, and let $\beta^{t}$ be the associated family of piecewise geodesic loops. Then there is a closed geodesic $\alpha$ so that $\beta^{t} \rightarrow \alpha$.

As an improvement of Corollary 8.2, we have the following result, which states that if $\beta^{t}$ gets close enough to a geodesic along which all sectional curvatures are negative, then $\beta^{t}$ converges to that geodesic:

Proposition 8.7. Let $M$ be a Riemannian manifold with $\operatorname{inj}(M)>0$, and let $\alpha$ be a closed geodesic such that all sectional curvatures are negative at each point of the image of $\alpha$. Fix $\varepsilon>0$. Then there is a $\delta>0$ so that, if $d\left(b_{i}(0), b_{i+1}(0)\right)<\operatorname{inj}(M)-\varepsilon$ 
for all $i$ and $\beta^{t_{0}}$ is uniformly $\delta$-close to $\alpha \circ T_{x}$ for some $t_{0}>0$ and some $x \in \mathbb{R} / \mathbb{Z}$, then $\beta^{t} \rightarrow \alpha$ as $t \rightarrow \infty$.

Proof. Take $\delta$ small enough so that:

(i) if $p_{1}, p_{2} \in \bar{N}_{\delta}(\alpha)$ and $d\left(p_{1}, p_{2}\right)<\operatorname{inj}(M)-\varepsilon$, then $\left[p_{1}, p_{2}\right] \subset \bar{N}_{\delta}(\alpha)$;

(ii) $\bar{N}_{\delta}(\alpha)$ is a closed manifold with boundary, smoothly embedded in $M$;

(iii) any loop uniformly $\delta$-close to $\alpha \circ T_{x}$ for some $x \in \mathbb{R} / \mathbb{Z}$ is homotopic to $\alpha$ through a family of loops in $\bar{N}_{\delta}(\alpha)$;

(iv) any closed geodesic $\gamma$ in $\bar{N}_{\delta}(\alpha)$ homotopic to $\alpha$ through a family of loops in $\bar{N}_{\delta}(\alpha)$ differs from $\alpha$ by a rotation in the domain.

For condition (iv), we argue on general grounds that taking $\delta$ small forces $\gamma$ to be uniformly close to $\alpha \circ T_{x}$ for some $x \in S^{1}$, and then we use the fact that a closed geodesic on $M$ along which all sectional curvatures are negative is isolated in the space of closed geodesics on $M$; the argument is straightforward, and we omit the details. For (i), we use Lemma 8.4. For (ii) and (iii), we take the image under exp of a neighborhood of the zero section in the normal bundle of $\alpha\left(S^{1}\right)$.

Suppose we have initial conditions for pursuit $\left\{b_{i}(0)\right\}_{i \in \mathbb{Z} / n}$ with relative distances $d\left(b_{i}(0), b_{i+1}(0)\right)<\operatorname{inj}(M)-\varepsilon$ for all $i$, and the associated piecewise geodesic loop $\beta^{t_{0}}$ is uniformly $\delta$-close to $\alpha \circ T_{x}$ for some $t_{0}>0, x \in \mathbb{R} / \mathbb{Z}$. By (i), (ii), and Proposition 5.1, we have $\beta^{t} \subset \bar{N}_{\delta}(\alpha)$ for all $t \geq t_{0}$. Using Proposition 6.1, we get a sequence $t_{j} \rightarrow \infty$ and a geodesic $\gamma$ contained in $\bar{N}_{\delta}(\alpha)$ so that $\beta^{t_{j}} \rightarrow \gamma$ uniformly. So $\beta^{t_{0}}$ is homotopic through a family of loops in $\bar{N}_{\delta}(\alpha)$ to $\gamma$. But by (iii), $\beta^{t_{0}}$ is also homotopic through a family of loops in $\bar{N}_{\delta}(\alpha)$ to $\alpha$. Now by (iv), $\gamma$ differs from $\alpha$ by a rotation in the domain. So by Corollary 8.2, $\beta^{t} \rightarrow \alpha$ as $t \rightarrow \infty$.

\section{Appendix}

We prove a result (Corollary A.2 below) needed for the proof of Proposition 5.2.

Proposition A.1. Let $\left(M^{n}, g\right)$ be a Riemannian manifold, $p$ a point in $M,\left(U, x^{i}\right)$ a normal coordinate neighborhood centered at $p$. Let $\|\cdot\|$ be the Euclidean norm on $\left(U, x^{i}\right)$. Then for every $\varepsilon>0$, there is an $r$ such that $B_{r}(p) \subset U$ and for every geodesic $\gamma:[0, a] \rightarrow B_{r}(p)$,

for all $t \in(0, a]$.

$$
\left\|\frac{\dot{\gamma}(0)}{\|\dot{\gamma}(0)\|}-\frac{\gamma(t)-\gamma(0)}{\|\gamma(t)-\gamma(0)\|}\right\|<\varepsilon,
$$

Proof. Fix $\varepsilon>0$. Let $V$ be an open neighborhood of $p$ with closure contained in $U$. Then the Christoffel symbols $\Gamma_{i j}^{k}$ associated to $\left(U, x^{i}\right)$ are bounded on $V$. Find $\mu$ so that $\left|\Gamma_{i j}^{k}\right|<\mu$ on $V$ for all $i, j, k$. Take $r$ small enough that $B_{r}(p) \subset V$. Since $g_{i j}(p)=\delta_{i j}$, we may take $r$ small enough that any vector in $T B_{r}(p)$ of unit length 
with respect to $g$ has length less than 2 with respect to the Euclidean norm. Now, if $\gamma:[0, a] \rightarrow B_{r}(p)$ is a unit speed geodesic, we have

$$
\frac{d^{2} \gamma^{k}}{d t^{2}}=-\Gamma_{i j}^{k} \frac{d \gamma^{i}}{d t} \frac{d \gamma^{j}}{d t}
$$

so $\left|\frac{d^{2} \gamma^{k}}{d t^{2}}\right|<4 \mu n^{2}$; thus $\left\|\frac{d^{2} \gamma}{d t^{2}}\right\|<4 \mu n^{\frac{5}{2}}$. Integrating, we find for each $t \in[0, a]$,

$$
\|\dot{\gamma}(t)-\dot{\gamma}(0)\|<4 t \mu n^{\frac{5}{2}} \leq 8 r \mu n^{\frac{5}{2}}
$$

so taking $r$ smaller than $\left[8 \mu n^{\frac{5}{2}}\right]^{-1} \varepsilon$,

$$
\|\dot{\gamma}(t)-\dot{\gamma}(0)\|<\varepsilon .
$$

Integrating again, we get $\|\gamma(t)-\gamma(0)-t \dot{\gamma}(0)\|<t \varepsilon$, so

$$
\left\|\frac{\gamma(t)-\gamma(0)}{t}-\dot{\gamma}(0)\right\|<\varepsilon
$$

for $t \in(0, a]$. Assuming $r$ is chosen small enough so that any $v \in T B_{r}(p)$ with $g(v, v)=1$ satisfies $\|v-v /\| v\|\|<\varepsilon$, we have

$$
\left\|\frac{\gamma(t)-\gamma(0)}{t}-\frac{\dot{\gamma}(0)}{\|\dot{\gamma}(0)\|}\right\|<2 \varepsilon
$$

Hence, the Euclidean distance from the unit vector $\dot{\gamma}(0) /\|\dot{\gamma}(0)\|$ to the line spanned by $\gamma(t)-\gamma(0)$ is less than $2 \varepsilon$. For $\varepsilon$ small enough, this implies

$$
\left\|\frac{\gamma(t)-\gamma(0)}{\|\gamma(t)-\gamma(0)\|}-\frac{\dot{\gamma}(0)}{\|\dot{\gamma}(0)\|}\right\|<3 \varepsilon
$$

With notation as in the last proposition, we have the following:

Corollary A.2. For every $\varepsilon>0$, there is an $r$ so that $B_{r}(p) \subset U$ and for any two geodesics $\gamma_{1}:\left[0, a_{1}\right] \rightarrow B_{r}(p)$ and $\gamma_{2}:\left[0, a_{2}\right] \rightarrow B_{r}(p)$ with $\gamma_{1}(0)=\gamma_{2}(0)$, the metric angle between $\dot{\gamma}_{1}(0)$ and $\dot{\gamma}_{2}(0)$ is within $\varepsilon$ of the Euclidean angle between $\gamma_{1}\left(a_{1}\right)-\gamma_{1}(0)$ and $\gamma_{2}\left(a_{2}\right)-\gamma_{2}(0)$.

Proof. By the last part and uniform continuity of the spherical distance function $S^{n-1} \times S^{n-1} \rightarrow \mathbb{R}$ on the unit sphere, we can choose $r$ small enough that the Euclidean angle between the vectors $\gamma_{1}\left(a_{1}\right)-\gamma_{1}(0)$ and $\gamma_{2}\left(a_{2}\right)-\gamma_{2}(0)$ is within $\frac{\varepsilon}{2}$ of the Euclidean angle between $\dot{\gamma}_{1}(0)$ and $\dot{\gamma}_{2}(0)$, for any two unit speed geodesics $\gamma_{1}:\left[0, a_{1}\right] \rightarrow B_{r}(p)$ and $\gamma_{2}:\left[0, a_{2}\right] \rightarrow B_{r}(p)$ with $\gamma_{1}(0)=\gamma_{2}(0)$. Then, if necessary, we choose $r$ smaller so that for any two vectors $u, v \in T B_{r}(p)$ based at the same point, the Euclidean angle between $u$ and $v$ is within $\frac{\varepsilon}{2}$ of the metric angle. 


\section{Acknowledgements}

This research was conducted mostly at the SUMMER@ICERM Undergraduate Summer Research Program in 2012. I would like to thank Tarik Aougab and Sergei Tabachnikov for their mentorship. I would like to thank Francisc Bozgan for pointing out the application of Jensen's inequality in Section 3. I would like to thank Anton Petrunin for a MathOverflow answer which helped with the proof of Proposition 8.1.

\section{References}

[Behroozi and Gagnon 1979] F. Behroozi and R. Gagnon, "Cyclic pursuit in a plane", J. Math. Phys. 20:11 (1979), 2212-2216. MR Zbl

[Borsuk 1947] K. Borsuk, "Sur la courbure totale des courbes fermées", Ann. Soc. Polon. Math. 20 (1947), 251-265. MR

[Lucas 1877] E. Lucas, “Questions proposées, $n^{\circ} 251$ ”, pp. 175-176 in Nouvelle correspondance mathématique, vol. 3, edited by E. Catalan et al., F. Hayez, Brussels, 1877.

[Richardson 2001a] T. Richardson, "Stable polygons of cyclic pursuit", Ann. Math. Artif. Intell. 31:1-4 (2001), 147-172. MR

[Richardson 2001b] T. J. Richardson, "Non-mutual captures in cyclic pursuit", Ann. Math. Artif. Intell. 31:1-4 (2001), 127-146. MR

Received October 4, 2016. Revised December 18, 2016.

DMITRI GEKHTMAN

DEPARTMENT OF MATHEMATICS

CALIFORNiA INSTITUTE OF TECHNOLOGY

PASADENA, CA 91125

UNITED STATES

dgekhtma@caltech.edu 


\title{
PACIFIC JOURNAL OF MATHEMATICS
}

Founded in 1951 by E. F. Beckenbach (1906-1982) and F. Wolf (1904-1989)

$$
\text { msp.org/pjm }
$$

\section{EDITORS}

\author{
Don Blasius (Managing Editor) \\ Department of Mathematics \\ University of California \\ Los Angeles, CA 90095-1555 \\ blasius@math.ucla.edu
}

\author{
Paul Balmer \\ Department of Mathematics \\ University of California \\ Los Angeles, CA 90095-1555 \\ balmer@math.ucla.edu \\ Robert Finn \\ Department of Mathematics \\ Stanford University \\ Stanford, CA 94305-2125 \\ finn@math.stanford.edu \\ Sorin Popa \\ Department of Mathematics \\ University of California \\ Los Angeles, CA 90095-1555 \\ popa@math.ucla.edu
}

\author{
Vyjayanthi Chari \\ Department of Mathematics \\ University of California \\ Riverside, CA 92521-0135 \\ chari@math.ucr.edu \\ Kefeng Liu \\ Department of Mathematics \\ University of California \\ Los Angeles, CA 90095-1555 \\ liu@math.ucla.edu \\ Igor Pak \\ Department of Mathematics \\ University of California \\ Los Angeles, CA 90095-1555 \\ pak.pjm@gmail.com \\ Paul Yang \\ Department of Mathematics \\ Princeton University \\ Princeton NJ 08544-1000 \\ yang@math.princeton.edu
}

\section{PRODUCTION}

Silvio Levy, Scientific Editor, production@msp.org

\section{SUPPORTING INSTITUTIONS}

ACADEMIA SINICA, TAIPEI

CALIFORNIA INST. OF TECHNOLOGY

STANFORD UNIVERSITY

UNIV. OF BRITISH COLUMBIA

UNIV. OF CALIFORNIA, BERKELEY

UNIV. OF CALIFORNIA, DAVIS

UNIV. OF CALIFORNIA, LOS ANGELES

UNIV. OF CALIFORNIA, RIVERSIDE

UNIV. OF CALIFORNIA, SAN DIEGO

UNIV. OF CALIF., SANTA BARBARA
KEIO UNIVERSITY

MATH. SCIENCES RESEARCH INSTITUTE

NEW MEXICO STATE UNIV.

OREGON STATE UNIV.
Daryl Cooper

Department of Mathematics

University of California

Santa Barbara, CA 93106-3080 cooper@math.ucsb.edu

Jiang-Hua Lu

Department of Mathematics

The University of Hong Kong

Pokfulam Rd., Hong Kong

jhlu@maths.hku.hk

$$
\text { Jie Qing }
$$

Department of Mathematics

University of California

Santa Cruz, CA 95064

qing@cats.ucsc.edu
UNIV. OF CALIF., SANTA CRUZ

UNIV. OF MONTANA

UNIV. OF OREGON

UNIV. OF SOUTHERN CALIFORNIA

UNIV. OF UTAH

UNIV. OF WASHINGTON

WASHINGTON STATE UNIVERSITY

These supporting institutions contribute to the cost of publication of this Journal, but they are not owners or publishers and have no responsibility for its contents or policies.

See inside back cover or msp.org/pjm for submission instructions.

The subscription price for 2017 is US \$450/year for the electronic version, and \$625/year for print and electronic.

Subscriptions, requests for back issues and changes of subscriber address should be sent to Pacific Journal of Mathematics, P.O. Box 4163, Berkeley, CA 94704-0163, U.S.A. The Pacific Journal of Mathematics is indexed by Mathematical Reviews, Zentralblatt MATH, PASCAL CNRS Index, Referativnyi Zhurnal, Current Mathematical Publications and Web of Knowledge (Science Citation Index).

The Pacific Journal of Mathematics (ISSN 0030-8730) at the University of California, c/o Department of Mathematics, 798 Evans Hall \#3840, Berkeley, CA 94720-3840, is published twelve times a year. Periodical rate postage paid at Berkeley, CA 94704, and additional mailing offices. POSTMASTER: send address changes to Pacific Journal of Mathematics, P.O. Box 4163, Berkeley, CA 94704-0163.

PJM peer review and production are managed by EditFLOW ${ }^{\circledR}$ from Mathematical Sciences Publishers.

PUBLISHED BY

\section{I. mathematical sciences publishers}

nonprofit scientific publishing

http://msp.org/

(C) 2017 Mathematical Sciences Publishers 


\section{PACIFIC JOURNAL OF MATHEMATICS}

Volume $289 \quad$ No. $1 \quad$ July 2017

Restricted Poisson algebras

YAN-Hong BAO, YU YE and JAMES J. ZHANG

Remarks on GJMS operator of order six

Xuezhang Chen and Fei Hou

On the asymptotic behavior of Bergman kernels for positive line bundles

TIEn-CUOng Dinh, XiAONAN MA and Viêt-AnH NGUYÊN

Molino theory for matchbox manifolds

Jessica Dyer, Steven HuRder and Olga LuKina

Cyclic pursuit on compact manifolds

DMITRI GEKHTMAN

Criticality of the axially symmetric Navier-Stokes equations

ZHEN LEI and QI S. ZHANG

Convexity of the entropy of positive solutions to the heat equation on quaternionic contact and CR manifolds

DIMITER VASSILEV

On handlebody structures of rational balls

LUKE WILLIAMS

On certain Fourier coefficients of Eisenstein series on $G_{2}$ 